\title{
INTENSIDAD DE APOYOS, SALUD MENTAL, EMPLEO Y SU RELACIÓN CON RESULTADOS DE CALIDAD DE VIDA
}

\section{Intensity of supports, mental bealth, employment and its relationship with results of quality of life}

Juan Antonio González Aguilar Asociación en favor de las personas con discapacidad intelectual de Córdoba (Aprosub). C/ Poeta Juan Ramón Jiménez, 8-Bis. 14012 Córdoba

juangonzalez@aprosub.es

Recepción: 6 de noviembre de 2017

Aceptación definitiva: 5 de diciembre de 2018

Resumen: La mejora de resultados personales y en la calidad de vida de las personas con discapacidad intelectual se ha convertido en el principal reto de las organizaciones prestadoras de apoyos. Aprosub incorpora en el 2010 la evaluación de calidad de vida como un resultado clave de la organización. Este artículo muestra los resultados en términos de calidad de vida y su relación con diferentes variables consideradas relevantes como la intensidad de apoyos, diagnósticos de salud mental y medicación psiquiátrica. Del mismo modo se valora la relación de programas inclusivos como el programa de empleo con apoyo desarrollado en la entidad con resultados de calidad de vida. Los datos contemplados se han obtenido mediante las escalas INICO-FEAPs y San Martín en función de las necesidades de apoyo. Corresponden a 412 personas adultas con discapacidad intelectual, de las cuales el $39,3 \%$ son mujeres y el $60,7 \%$ hombres. A todas ellas se les prestan apoyos en servicios de Aprosub en la provincia de Córdoba. Los resultados obtenidos muestran la relación positiva, en términos de calidad de vida, de programas inclusivos como el de empleo con apoyo. Igualmente, se ha detectado que hay una relación directa entre las necesidades de apoyo de las personas y sus resultados de calidad de vida. Por otra parte, los datos muestran que la presencia de algún trastorno de salud mental hace que las puntuaciones de calidad de vida sean menores que en el caso de personas que no padecen el trastorno.

Palabras Clave: apoyos; salud mental; empleo; calidad de vida. 
АвsтRAст: The improvement of personal results and quality of life for the people with intellectual disability has become the main challenge for the organizations destined to provide assistance. Aprosub incorporated in 2010 the evaluation of quality of life as a key result for the organization. This article displays the results in terms of quality of life and its relationship with different variables considered relevant as the intensity of aids, mental health diagnosis and psychiatric medication. Likewise, the impact of inclusive agendas, such as the program of employment with support developed in the entity, is taken into consideration. The data provided was obtained through the scales INICO-FEAPS y San Martín according to the assistance needs. The data refers to 412 adults with intellectual disability, of which $39,3 \%$ are women and $60,7 \%$ are men. All of them are provided with support in Aprosub services in the province of Cordoba. The results obtained show the positive impact, in terms of quality of life, of inclusive programs as the employment with support program. Furthermore, we have found a direct relationship between the needs for assistance of one person and its results in quality of life. Conversely, the data shows that the presence of any mental health issue decreases the punctuation of quality of life in comparison with the individuals that do not endure the condition.

KEY wORDs: supports; mental health; employment; quality of life.

\title{
1. Introducción
}

CTUalmente Calidad de Vida se ha convertido en la principal referencia como resultado de las prácticas centradas en las personas que se desarrollan en las organizaciones prestadoras de apoyo a las personas con discapacidad intelectual.
\end{abstract}

Schalock y Verdugo (2003) nos plantean que pueden existir más de cien definiciones del término "Calidad de Vida”. Por lo que su propuesta es intentar hacer "una descripción operativa del término y de su significado" (Schalock y Verdugo, 2003).

Otra de las cuestiones clave a la hora de contemplar el constructo es valorar si este corresponde más a un concepto subjetivo, es decir, solo valorable por la propia persona, o ser considerado como algo objetivo y valorable por agentes externos. De igual manera para referirnos a calidad de vida no solo tendríamos que fijarnos en componentes internos, sino que hay que entenderlo desde una perspectiva integradora, en la que se contemplen tanto los elementos internos como la fuerte influencia que los contextos pueden tener en los individuos. Este aspecto cobra especial importancia a la hora de analizar los datos obtenidos y ponderar los mismos en relación a si la valoración proviene de la propia persona o de un evaluador externo.

Las dificultades para definir el significado exacto de Calidad de Vida han llevado a los autores (Schalock y Verdugo, 2002) a realizar un planteamiento en base a dimensiones y no una descripción del mismo. Concretamente son ocho las dimensiones identificadas en el modelo: Bienestar emocional, Relaciones interpersonales, Bienestar material, Desarrollo personal, Bienestar físico, Autodeterminación, Inclusión social y Derechos. 
Por otra parte, el paradigma de apoyos se ha convertido en otro de los elementos clave a la hora de planificar el trabajo en el ámbito de las personas con discapacidad intelectual. Especialmente relevante ha sido la evolución del modelo de prestación de apoyos pasando de un modelo centrado en el servicio a un modelo centrado en la persona. Este cambio ha venido reforzado por el enfoque de clasificación de los apoyos en función de su intensidad relegando la postura clásica de clasificar a las personas atendiendo a sus capacidades. Igualmente, la aparición de una escala como la Escala de Intensidad de Apoyos (sIs), adaptada a la población española (Verdugo, Arias e Ibáñez, 2007), ha dado el impulso definitivo para contemplar los apoyos y su intensidad como elementos relevantes en los procesos de atención a personas.

Uno de los objetivos que este estudio se propone es identificar las diferencias de resultados en calidad de vida en función de la intensidad de apoyo de las personas, en este caso usaremos la escala sis para medir intensidad de apoyos y las escalas InicoFeaps (Verdugo et al., 2013) y San Martín (Verdugo et al., 2014), para evaluar calidad de vida.

Otro de los aspectos relevantes que abordamos en este artículo es la posible relación entre diagnósticos de salud mental, el uso de medicación psiquiátrica y su influencia en los resultados de calidad de vida.

Por último, plasmaremos las diferencias existentes en términos de calidad de vida entre personas que participan en el programa de empleo con apoyo y las que no participan.

\subsection{Calidad de Vida e Intensidad de Apoyos}

Desde la revisión del concepto de discapacidad intelectual realizada por la AIDD en 1992 (Luckasson et al., 1992), el conocido como paradigma de los apoyos ha tenido una gran repercusión en los enfoques y prácticas de trabajo desarrolladas en las organizaciones. Esta puede venir definida por varios aspectos:

- Pasamos de clasificar a las personas para poner el acento en los apoyos que esta necesita y la intensidad de los mismos.

- El poner el acento en los apoyos y no en las limitaciones de las personas permite obtener un prisma más amplio para planificar el trabajo.

- El contexto se convierte en un elemento clave a contemplar, los apoyos también están en función del contexto y de los diferentes sistemas del mismo.

- Se han incorporado herramientas que evalúan la intensidad de los apoyos, como la Escala sis, lo que nos permite afinar mucho más en el ámbito de intervención.

Este planteamiento nos permite enfocar los programas y actividades desde una perspectiva distinta, dejando de poner el acento en lo que no puede hacer una persona y poniendo el foco en qué apoyos necesitaba para poder hacer algo. Aun así, se seguían perdiendo de vista algunos aspectos importantes como ¿qué relación tenían los aprendizajes en la vida de las personas? Es decir, los apoyos prestados a las personas para el aprendizaje o desarrollo de actividades suponía una mejora real en la vida de estas o no. 
A partir de los primeros años de este siglo, Schalock y Verdugo (2002) comienzan a desarrollar un nueva teoría en el ámbito de la calidad de vida, describiendo dimensiones, indicadores, ámbitos de actuación, evaluación, etc., lo que nos permite dar un nuevo giro en las estrategias de trabajo y orientar los resultados de las prácticas hacia un impacto real en la vida de las personas.

Tomamos como punto de partida la premisa de que cualquier persona puede mejorar su vida si obtiene los apoyos necesarios en las áreas que son fundamentales para ella. Para ello es primordial conocer la intensidad de esos apoyos y las áreas relevantes para las personas. $Y$ es aquí donde nos encontramos con un dilema que para nosotros es de gran importancia: ¿qué ocurre con las personas con altas necesidades de apoyo en la mayoría de áreas? Es por ello que hemos querido aprovechar este estudio para identificar si hay algún tipo de relación entre las necesidades de apoyos y los resultados en términos de calidad de vida.

\subsection{Calidad de Vida y Salud Mental}

La salud mental de las personas con discapacidad intelectual se ha convertido, junto a los procesos de envejecimiento, en uno de los retos más importantes para las organizaciones prestadoras de apoyo. Especialmente difícil es el abordaje de los problemas de salud mental, ya que su identificación y diagnóstico se complican de sobremanera debido principalmente al efecto eclipsador que supone la discapacidad intelectual. Se estima (Carulla, 2007) que aproximadamente el 30\% de las personas con discapacidad intelectual presentan algún problema de salud mental. Si nos fijamos en trastornos concretos, aproximadamente el $10 \%$ presentan trastornos psicóticos, el $8.6 \%$ trastornos del humor y en torno al $30 \%$ trastornos de conducta. No obstante, como indicábamos anteriormente, el diagnóstico es muy difícil en muchos de los casos y especialmente complejo en el caso de personas con altas necesidades de apoyo.

En nuestro estudio hemos querido analizar las posibles diferencias que existen en términos de calidad de vida, comparando resultados entre personas con diagnóstico de enfermedad mental y aquellas que no lo tienen. Igualmente, analizamos las diferencias entre aquellas personas que usan medicación y las que no.

\subsection{Calidad de Vida y Empleo}

Desde hace décadas el empleo con apoyo se ha mostrado como la mejor opción para potenciar el empleo de personas con discapacidad intelectual en contextos comunitarios. No obstante, el acceder al mercado de trabajo sigue suponiendo una importante dificultad para personas con discapacidad teniendo un alto impacto en algunas dimensiones de calidad de vida como Inclusión Social, Desarrollo Personal, Autodeterminación y Derechos (Verdugo, 2006).

En nuestro trabajo hemos querido comparar las relaciones existentes en términos de calidad de vida entre personas que participan en programas de empleo frente a personas 
que no participan en estos programas. Y, por otra, parte conocer si hay diferencias entre las personas que participan en el programa y aquellas que consiguen un empleo.

\section{Método}

\subsection{Participantes}

Los datos que se han utilizado en este estudio corresponden a toda la población adulta de personas con discapacidad intelectual a las que se prestan apoyos en los servicios de Aprosub. Esto supone que los datos globales usados corresponden a 412 personas, de las cuales el 39,3\% son mujeres y el 60,7\% son hombres. La media de edad ha sido de 44,85 años, siendo muy similar entre el grupo de hombres $(45,70)$ y el de mujeres $(43,48)$.

Aprosub cuenta con seis centros en que presta servicios a personas adultas en diferentes poblaciones de la provincia de Córdoba. La distribución por poblaciones ha sido la siguiente: Baena 16,7\%, Castro del Río 12,10\%, Córdoba 41,30\%, Montilla 14,10\%, Palma del Río 11,20\% y Peñarroya 4,6\%.

$\mathrm{Si}$ atendemos a la distribución por servicios, el 51,5\% asisten a servicios ocupacionales, el 11,4\% a servicios de día, el 31,8\% a residencias de adultos ${ }^{1}$ y el 5,3\% a servicios residenciales para personas con altas necesidades de apoyo.

En cuanto a la puntuación media de los índices de intensidad de apoyos (sis) de las personas que asisten a servicios ocupacionales es de 97,08, similar al de las personas que viven en servicios residenciales, 95,53. En el caso de las personas que asisten a servicios para personas con más necesidades de apoyo, las puntuaciones medias son de 105,31 en residencia y 109,54 en servicios de día.

Atendiendo a los dos parámetros planteados en relación con la salud mental, diagnóstico de enfermedad mental y medicación psiquiátrica, reseñar que el 20,40\% de las personas tienen algún diagnóstico de enfermedad mental, mientras que al 34,5\% se les administra medicación psiquiátrica. Esta diferencia, entendemos que se puede deber a que a veces se administra medicación de este tipo sin haber finalizado un proceso de diagnosis o en ocasiones sin ni siquiera iniciarlo. Consideramos que este es un aspecto de suma importancia en el que sin duda hay que profundizar.

Por último, referenciar que el 18,4\% están participando en el programa de empleo con apoyo que desarrolla la entidad. Todas estas personas están en servicios ocupacionales o residenciales para personas con necesidades de apoyo limitado o intermitente.

\subsection{Instrumentos}

Desde el año 2010 hasta la actualidad, en Aprosub, hemos usado diversas escalas para la evaluación de calidad de vida. Hasta la aparición de la escala Inico-Feaps en

Las personas que asisten a servicios residenciales también asisten a servicios de día con terapia ocupacional. En este estudio se han contemplado sin repetir los casos, es decir, las personas que van a servicios residenciales no se han contemplado en el grupo de servicios de día. 
2013 (Verdugo et al., 2013), usábamos la Escala Integral de Calidad de Vida (Verdugo, Gómez, Arias y Schalock, 2009) y la Escala Gencat (Verdugo, Arias, Gómez y Schalock, 2009) en el caso de personas que no podían responder a un cuestionario autoadministrado. Es a partir del año 2014 cuando en la entidad se sustituyen estas dos por la Escala Inico-Feaps y la Escala San Martín (Verdugo, Gómez, Arias, Santamaría, Navallas, Fernández e Hierro, 2014), usada de manera específica para personas con altas necesidades de apoyo.

\subsection{Procedimiento}

En el año 2010 Aprosub incorporó como resultado clave de la organización el impacto en calidad de vida de las personas a las que se les presta apoyo en sus servicios. Hasta el año 2014 la recogida de datos se realizaba con carácter bienal, ya que no terminábamos de identificar con claridad si los resultados podrían ser relevantes si se miden en un periodo menor de tiempo. En ese tiempo observamos que podía haber acontecimientos importantes para las personas que tuviesen un impacto directo en la evaluación de calidad de vida y que posteriormente ese impacto desapareciese, por lo que optamos por realizar una evaluación anual entre los meses de noviembre y diciembre de cada año. Las puntuaciones utilizadas en este artículo son las correspondientes a las 412 personas, obtenidas en los años 2015 y 2016.

\subsection{Análisis de datos}

En relación a las variables ${ }^{2}$ usadas en el estudio hemos tomado como referencia los índices de calidad de vida arrojados por las escalas Inico-Feaps y San Martín, tanto desde la perspectiva de autoevaluación como de evaluación externa en los años 2015 y 2016. Atendiendo a estos datos hemos optado por usar como variable la media de los años, al considerarla más robusta desde el punto de vista analítico y tras comprobar, con la prueba de Kolmogorov-Smirnov, que en ambos casos no podíamos descartar que las muestras proviniesen de distribuciones diferentes con una significación del $5 \%$ (para puntuaciones externas $\mathrm{p}=0,55$ y puntuaciones autoevaluación $\mathrm{p}=0,52$ ).

Por otra parte, hemos constatado que, tanto en el caso de las puntuaciones obtenidas con la Escala Inico-Feaps, como las obtenidas con la Escala San Martín, la distribución de la muestra cumple con los criterios de normalidad con un $95 \%$ de confianza, tanto en las pruebas de Kolmogorov-Smirnov, como con la prueba w de Shapiro-wilk.

Para el análisis de los datos hemos usado el programa de análisis estadístico Statgraphics Centurion xv para Windows.

2 Denominación de las variables: ICVAT15 = Índice de Calidad de Vida, prueba autoadministrada, 2015; ICVAT16 = Índice de Calidad de Vida, prueba autoadministrada, 2016; ICVAT15-16 = Índice de Calidad de Vida, prueba autoadministrada, media de 2015 y 2016; ICveXT15 = Índice de Calidad de Vida, prueba evaluador externo, 2015; ICVEXT16 = Índice de Calidad de Vida, prueba evaluador externo, 2016; ICVEXT15-16 = Índice de Calidad de Vida, prueba evaluador externo, media de 2015 y 2016. 


\section{Resultados}

Los resultados serán presentados atendiendo a tres bloques: Calidad de Vida e Intensidad de Apoyos; Calidad de Vida y Salud Mental, y, por último, Calidad de Vida y Empleo.

Tal y como se ha comentado con anterioridad, los datos se refieren a los años 2015 y 2016, ya que, aunque contamos con datos desde el año 2010, el uso de diferentes escalas en este periodo dificulta la interpretación de los resultados. En este sentido remarcar que los datos se han obtenido con la Escala Inico-Feaps y la Escala San Martín, en el caso de personas que no pudiesen cumplimentar una escala de autopercepción.

\subsection{Calidad de Vida e Intensidad de Apoyos}

Como se ha referido con antelación nos planteamos analizar la relación que se establece entre la intensidad de apoyos, obtenida con la Escala sis, y los resultados de calidad de vida, obtenidos con la Escala Inico-Feaps y la Escala San Martín. Hemos optado por utilizar los datos de las escalas por separado para evitar generar confusión en los resultados, ya que, en el caso de la Escala San Martín, no se contempla la puntuación obtenida de manera directa por parte de la propia persona.

Podemos observar (Tabla 1) que tanto en el índice de calidad de vida aportado por datos autoevaluados como por el evaluador externo, existe una correlación directa negativa entre las puntuaciones obtenidas en la Escala sis y los resultados en calidad de vida. Es decir, a más intensidad de apoyos peores resultados en calidad de vida y viceversa.

\begin{tabular}{|l|l|c|c|c|c|}
\hline \multicolumn{7}{|c|}{ TABLA 1. Correlaciones I } \\
\hline $\begin{array}{l}\text { Datos obtenidos con la } \\
\text { Escala Inico-Feaps }\end{array}$ & $\begin{array}{c}\text { Índice Calidad } \\
\text { de Vida 2015 } \\
\text { (Autoevaluación) }\end{array}$ & $\begin{array}{c}\text { Índice Calidad } \\
\text { de Vida 2016 } \\
\text { (Autoevaluación) }\end{array}$ & $\begin{array}{c}\text { Índice Calidad } \\
\text { de Vida 2015 } \\
\text { (Evaluador externo) }\end{array}$ & $\begin{array}{c}\text { Índice Calidad } \\
\text { de Vida 2016 } \\
\text { (Evaluador externo) }\end{array}$ \\
\hline SIS & Correlación de Person &,- 404 &,$- 333^{*}$ &,$- 219^{* * *}$ &,$- 339^{* *}$ \\
\hline & Sig. bilateral &, 000 &, 000 &, 000 &, 000 \\
\hline
\end{tabular}

* La correlación es significativa al nivel 0,01 (bilateral).

\begin{tabular}{|c|c|c|c|}
\hline \multicolumn{4}{|c|}{ TABLA 2. Correlaciones II } \\
\hline \multicolumn{2}{|r|}{ Datos obtenidos con la Escala San Martín } & $\begin{array}{c}\text { Índice Calidad } \\
\text { de Vida } 2015 \\
\text { (Evaluador externo) }\end{array}$ & $\begin{array}{c}\text { Índice Calidad } \\
\text { de Vida } 2016 \\
\text { (Evaluador externo) }\end{array}$ \\
\hline SIS & Correlación de Person &, $447^{* * *}$ &, $330^{* *}$ \\
\hline & Sig. bilateral &, 000 &, 000 \\
\hline
\end{tabular}

La correlación es significativa al nivel 0,01 (bilateral). 
No obstante, en los datos obtenidos con la Escala San Martín (Tabla 2) los resultados se han comportado de manera distinta, es decir, observamos que existe relación, pero esta se da en sentido positivo; a mayor necesidad de apoyos mayor puntuación en términos de calidad de vida.

Esta diferencia de comportamiento puede ser un elemento interesante a estudiar con muestras más amplias, ya que, a priori, podríamos entender que, a más necesidades de apoyo, menor calidad de vida, como en el caso anterior. Quizás podría estar relacionado con las características de la prueba utilizada, ya que la Escala San Martín solo contempla la evaluación desde la perspectiva de un evaluador externo. Además, si tenemos en cuenta las características de las personas evaluadas, altas necesidades de apoyo, podría suceder que unas expectativas más bajas incidan en una mejor percepción de la calidad de vida.

\subsection{Calidad de Vida y Salud Mental}

De las 412 personas que hemos contemplado en el estudio, 84 cuentan con un diagnóstico de salud mental, lo que supone un 20,4\% del total. Y a 142 se les administra medicación de carácter psiquiátrico, esto es un 34,5\%. Atendiendo a la clasificación por servicios indicada anteriormente, en los servicios para personas con altas necesidades de apoyo se atiende a 69 personas de las que el 11,6\% tienen diagnóstico en relación a su salud mental y se les administra medicación psiquiátrica al 40,6\%. En el caso de los servicios para personas con menos necesidades de apoyo, 343 personas, se han diagnosticado al 22,2\% y se administra medicación al 33,2\%.

En relación con los resultados de calidad de vida los presentaremos de manera diferenciada por cada uno de los grupos identificados, prestando atención al índice de calidad de vida, tanto desde una perspectiva externa como desde la perspectiva de autoevaluación. Igualmente, nos centraremos en los resultados sobre bienestar emocional, ya que es una dimensión relacionada directamente con parámetros de salud mental.

En primer lugar, presentamos (Tabla 3) los datos de personas a las que se prestan apoyos en residencias de adultos y servicios ocupacionales (menor intensidad de apoyos). Podemos observar que las personas que no cuentan con diagnóstico sobre enfermedad mental obtienen una mejor puntuación en términos globales de calidad de vida.

\begin{tabular}{|l|l|c|c|c|c|}
\hline \multicolumn{7}{|c|}{ Tabla 3. Salud Mental y menor intensidad de apoyos } \\
\hline \multirow{2}{*}{$\begin{array}{l}\text { Diagnóstico Salud } \\
\text { Mental }\end{array}$} & $\begin{array}{c}\text { Índice Calidad } \\
\text { de Vida 2015 } \\
\text { (Autoevaluación) }\end{array}$ & $\begin{array}{c}\text { Índice Calidad } \\
\text { de Vida 2016 } \\
\text { (Autoevaluación) }\end{array}$ & $\begin{array}{c}\text { Índice Calidad } \\
\text { de Vida 2015 } \\
\text { (Evaluador externo) }\end{array}$ & $\begin{array}{c}\text { Índice Calidad } \\
\text { de Vida 2016 } \\
\text { (Evaluador externo) }\end{array}$ \\
\hline \multirow{2}{*}{ Sí } & Media & 98,53 & 98,03 & 99,46 & 98,61 \\
\cline { 2 - 6 } & Desv. típ. & 12,67 & 15,91 & 13,28 & 15,17 \\
\hline \multirow{2}{*}{ No } & Media & 101,68 & 101,52 & 103,26 & 103,97 \\
\cline { 2 - 6 } & Desv. típ. & 14,41 & 13,49 & 13,69 & 13,16 \\
\hline \multirow{2}{*}{ Total } & Media & 100,97 & 100,77 & 102,45 & 102,77 \\
\cline { 2 - 6 } & Desv. típ. & 14,07 & 14,08 & 13,67 & 13,79 \\
\hline
\end{tabular}

(C) Ediciones Universidad de Salamanca / CC BY-NC-ND

Siglo Cero, vol. 50 (2), n. ${ }^{\circ} 270,2019$, abril-junio, pp. 73-88 
Como comentamos anteriormente, y con la intención de ganar en robustez analítica, hemos usado para análisis de varianza (ANOva) la variable obtenida de las medias de los dos años (2015 y 2016).

Al realizar un ANOva de esta variable podemos contrastar, con un nivel de confianza del 95\%, que, en el caso de la autoevaluación, la puntuación de calidad de vida no se ve influenciada por el hecho de tener un diagnóstico sobre salud mental $(\mathrm{F}=$ 2,77; $\mathrm{p}=0,09)$. No obstante, no ocurre lo mismo si prestamos atención a la puntuación procedente de una evaluación externa, en la que sí se aprecia dependencia entre las dos variables $(\mathrm{F}=7,28 ; \mathrm{p}=0,007)$. En este caso habría que analizar con más detalle la posible influencia del evaluador externo.

En cuanto a los resultados obtenidos con personas con altas necesidades de apoyo los datos no aportan una línea clara (Tabla 4).

\begin{tabular}{|c|c|c|c|}
\hline \multicolumn{4}{|c|}{ Tabla 4. Salud Mental y mayor intensidad de apoyos } \\
\hline \multicolumn{2}{|c|}{ Diagnóstico Salud Mental } & $\begin{array}{c}\text { Índice Calidad de Vida } 2015 \\
\text { (Evaluador externo) }\end{array}$ & $\begin{array}{c}\text { Índice Calidad de Vida } 2016 \\
\text { (Evaluador externo) }\end{array}$ \\
\hline \multirow{2}{*}{ Sí } & Media & 92,00 & 97,75 \\
\hline & Desv. típ. & 22,27 & 10,30 \\
\hline \multirow{2}{*}{ No } & Media & 83,53 & 100,38 \\
\hline & Desv. típ. & 31,54 & 12,34 \\
\hline \multirow{2}{*}{ Total } & Media & 84,76 & 100,01 \\
\hline & Desv. típ. & 30,35 & 12,02 \\
\hline
\end{tabular}

Al tener un número muy reducido de personas con diagnóstico sobre enfermedad mental (8 personas) hemos optado por no realizar ANOva ya que los resultados no serían relevantes.

Otro de los aspectos contemplados en nuestro análisis ha sido la comparación entre personas que toman medicación de carácter psiquiátrico respecto a las que no. Como hemos podido ver anteriormente el porcentaje de personas que toman medicación de este tipo es mayor que las que cuentan con un diagnóstico concreto de enfermedad mental. Esto nos sitúa en uno de los temas más controvertidos en relación con la salud mental y la discapacidad intelectual: el posible abuso de farmacología psiquiátrica en personas con discapacidad intelectual.

En cuanto a los resultados de calidad de vida podemos ver (Tabla 5), en el caso de personas con menos necesidades de apoyo, que se reproduce el mismo patrón que en el caso de diagnóstico de salud mental, es decir, aquellas personas a las que se administra medicación psiquiátrica obtienen una peor puntuación que a las que no se les administra.

Aunque de manera contraria a lo que ocurría en el caso de contemplar la variable "Diagnóstico de salud mental”, en esta ocasión, al realizar el ANOvA, podemos ver que, con un nivel de confianza del 95\%, sí hay una diferencia estadísticamente significativa en las puntuaciones de calidad de vida entre las personas que tienen tratamiento de medicación psiquiátrica y las que no. Esto ocurre tanto para los datos de autoevaluación, variable ICVAUT15-16 ( F = 5,80; $\mathrm{p}=0,0168)$, como para la evaluación externa, variable ICVEXT15-16 ( $\mathrm{F}=10,92 ; \mathrm{p}=0,0011)$. 


\begin{tabular}{|l|l|c|c|c|c|}
\hline \multicolumn{7}{|c|}{ Tabla 5. Medicación e impacto en calidad de vida } \\
\hline \multirow{2}{*}{ Uso de Medicación } & $\begin{array}{c}\text { Índice Calidad } \\
\text { de Vida 2015 } \\
\text { (Autoevaluación) }\end{array}$ & $\begin{array}{c}\text { Índice Calidad } \\
\text { de Vida 2016 } \\
\text { (Autoevaluación) }\end{array}$ & $\begin{array}{c}\text { Índice Calidad } \\
\text { de Vida 2015 } \\
\text { (Evaluador externo) }\end{array}$ & $\begin{array}{c}\text { Índice Calidad } \\
\text { de Vida 2016 } \\
\text { (Evaluador externo) }\end{array}$ \\
\hline \multirow{2}{*}{ Sí } & Media & 98,32 & 98,21 & 100,07 & 99,06 \\
\cline { 2 - 6 } & Desv. típ. & 13,63 & 15,43 & 13,66 & 14,31 \\
\hline \multirow{2}{*}{ No } & Media & 102,14 & 102,08 & 103,64 & 104,69 \\
\cline { 2 - 6 } & Desv. típ. & 14,10 & 13,31 & 13,56 & 13,16 \\
\hline \multirow{2}{*}{ Total } & Media & 100,96 & 100,84 & 102,50 & 102,81 \\
\cline { 2 - 6 } & Desv. típ. & 14,04 & 14,11 & 13,67 & 13,79 \\
\hline
\end{tabular}

No obstante, en el caso de personas con altas necesidades de apoyo, en esta ocasión la muestra es de 57 personas, no podemos establecer esa dependencia entre variables $(\mathrm{F}=0,19 ; \mathrm{p}=0,6650)$. Como comentábamos anteriormente este es un aspecto a estudiar con más detenimiento y valorar el posible efecto de la escala utilizada y la evaluación de agentes externos.

En cuanto al efecto concreto en la dimensión Bienestar Emocional (Tabla 6), como podría ser esperable, encontramos un patrón muy similar al anterior. Es decir, en todos los casos muestran mejores puntuaciones en Bienestar Emocional las personas que no han sido diagnosticadas en el ámbito de salud mental que las que sí lo han sido.

\begin{tabular}{|l|c|c|c|c|c|}
\hline \multicolumn{5}{|c|}{ TABLA 6. Impacto en la dimensión Bienestar Emocional } \\
\hline \multicolumn{2}{|l|}{} & \multicolumn{4}{|c|}{ Servicio Residencial Adultos / Servicio Ocupacional } \\
\hline $\begin{array}{l}\text { Diagnóstico de Salud } \\
\text { Mental }\end{array}$ & $\begin{array}{c}\text { B. Emocional 2015 } \\
\text { (Autoevaluación) }\end{array}$ & $\begin{array}{c}\text { B. Emocional 2015 } \\
\text { (Autoevaluación) }\end{array}$ & $\begin{array}{c}\text { B. Emocional 2015 } \\
\text { (Evaluador externo) }\end{array}$ & $\begin{array}{c}\text { B. Emocional 2016 } \\
\text { (Evaluador externo) }\end{array}$ \\
\hline \multirow{2}{*}{ Sí } & Media & 9,28 & 9,28 & 9,23 & 9,22 \\
\cline { 2 - 6 } & Desv. típ. & 2,71 & 3,26 & 2,72 & 2,98 \\
\hline \multirow{2}{*}{ No } & Media & 10,67 & 11,14 & 10,72 & 11,03 \\
\cline { 2 - 6 } & Desv. típ. & 2,62 & 2,65 & 2,63 & 2,58 \\
\hline \multirow{2}{*}{ Total } & Media & 10,36 & 10,72 & 10,42 & 10,64 \\
\cline { 2 - 6 } & Desv. típ. & 2,70 & 2,90 & 2,71 & 2,76 \\
\hline
\end{tabular}

De igual manera, al realizar un ANOva, podemos comprobar, con un nivel de confianza del 95\%, que existe una diferencia significativa en la dimensión Bienestar Emocional entre las personas diagnosticadas con algún trastorno de salud mental y las que no, tanto en los resultados procedentes de la autoevaluación $(\mathrm{F}=18,11 ; \mathrm{p}=$ $0,0000)$ como para la evaluación externa $(\mathrm{F}=30,00 ; \mathrm{p}=0,0000)$.

Este efecto es diferente al observado al contemplar los índices generales de calidad de vida, en el que no encontrábamos relación, lo que nos lleva a plantearnos que puede ser más interesante valorar los resultados en dimensiones concretas en función de la variable a estudiar más que acudir a los índices generales. 


\subsection{Calidad de Vida y Empleo}

La población de referencia para esta parte del estudio son personas a las que se presta apoyo en servicios ocupacionales o servicios residenciales para adultos. De las 343 personas que asisten a estos servicios, 76 participan en programas de empleo, lo que supone un $22,2 \%$. De estas 76 personas el $39,47 \%$ ha realizado prácticas en empresas ordinarias. Y el 15\% han conseguido un contrato.

Como es obvio, no todas las personas que asisten a un servicio ocupacional tienen la misma intensidad de apoyos. En nuestro caso, más del $63 \%$ obtienen una puntuación inferior a 96 en el índice de necesidades de apoyos de la sis, lo que supondría niveles de Apoyo i y iI, según la propia escala. Con la intención de minimizar el impacto de la variable "necesidades de apoyo", realizaremos las comparaciones solo en relación con las personas que tengan necesidades de apoyo parecidas a las que están participando en el programa de empleo. Así, la muestra total a analizar estará compuesta por 174 personas de servicios ocupacionales y residencias de adultos.

Si contemplamos los efectos en términos de calidad de vida, tomando el índice global de calidad de vida, tanto a nivel autoinforme como informe externo, podemos observar que la media de puntuaciones es más alta en personas que participan en el programa de empleo que en las que no participan. Esto ocurre tanto en las valoraciones realizadas por las propias personas como en el caso de evaluadores externos, como podemos ver en la tabla que mostramos a continuación (Tabla 7).

\begin{tabular}{|l|l|c|c|c|c|}
\hline \multicolumn{7}{|c|}{ Tabla 7. Empleo con Apoyo y Calidad de Vida } \\
\hline $\begin{array}{l}\text { Participación } \\
\text { en el programa } \\
\text { de empleo con apoyo }\end{array}$ & $\begin{array}{c}\text { Índice Calidad } \\
\text { de Vida 2015 } \\
\text { (Autoevaluación) }\end{array}$ & $\begin{array}{c}\text { Índice Calidad } \\
\text { de Vida 2016 } \\
\text { (Autoevaluación) }\end{array}$ & $\begin{array}{c}\text { Índice Calidad } \\
\text { de Vida 2015 } \\
\text { (Evaluador externo) }\end{array}$ & $\begin{array}{c}\text { Índice Calidad } \\
\text { de Vida 2016 } \\
\text { (Evaluador externo) }\end{array}$ \\
\hline \multirow{2}{*}{ Sí } & Media & 110,64 & 109,53 & 108,91 & 112,20 \\
\cline { 2 - 6 } & Desv. típ. & 11,26 & 10,77 & 12,62 & 10,14 \\
\hline \multirow{2}{*}{ No } & Media & 101,27 & 101,02 & 103,94 & 104,51 \\
\cline { 2 - 6 } & Desv. típ. & 14,38 & 14,85 & 13,88 & 14,00 \\
\hline \multirow{2}{*}{ Total } & Media & 105,03 & 104,25 & 105,83 & 107,33 \\
\cline { 2 - 6 } & Desv. típ. & 13,96 & 14,04 & 13,59 & 13,22 \\
\hline
\end{tabular}

Al realizar un ANOva, atendiendo a la variable obtenida de las medias de los dos años (ICVAUT15-16 e ICVEXT15-16), podemos ver que, con un nivel de confianza del $95 \%$, existen diferencias significativas entre las puntuaciones obtenidas por personas que participan en el programa de empleo con apoyo y las que no, tanto desde la perspectiva externa $(\mathrm{F}=25,58, \mathrm{p}=0,0000)$ como desde la autoevaluada $(\mathrm{F}=30,47$; $\mathrm{p}=0,0000)$.

Si tomamos los datos solo de las personas que están en el programa de empleo con apoyo y comparamos las puntuaciones de los índices de calidad de vida, de las personas que realizan prácticas y las que no, vemos que esas puntuaciones se igualan bastante (Tabla 8 ). 


\section{Tabla 8. Prácticas en empleo y Calidad de Vida}

\begin{tabular}{|l|l|c|c|c|c|}
\hline \multicolumn{2}{|l|}{$\begin{array}{l}\text { Participación en prácticas } \\
\text { de empleo }\end{array}$} & $\begin{array}{c}\text { Índice Calidad } \\
\text { de Vida 2015 } \\
\text { (Autoevaluación) }\end{array}$ & $\begin{array}{c}\text { Índice Calidad } \\
\text { de Vida 2016 } \\
\text { (Autoevaluación) }\end{array}$ & $\begin{array}{c}\text { Índice Calidad } \\
\text { de Vida 2015 } \\
\text { (Evaluador externo) }\end{array}$ & $\begin{array}{c}\text { Índice Calidad } \\
\text { de Vida 2016 } \\
\text { (Evaluador externo) }\end{array}$ \\
\hline \multirow{2}{*}{ Sí } & Media & 111,38 & 110,48 & 109,46 & 113,23 \\
\cline { 2 - 6 } & Desv. típ. & 11,41 & 9,43 & 13,03 & 9,88 \\
\hline \multirow{2}{*}{ No } & Media & 110,06 & 108,85 & 108,50 & 111,47 \\
\cline { 2 - 6 } & Desv. típ. & 11,29 & 11,72 & 12,48 & 10,40 \\
\hline \multirow{2}{*}{ Total } & Media & 110,64 & 109,53 & 108,91 & 112,20 \\
\cline { 2 - 6 } & Desv. típ. & 11,26 & 10,77 & 12,62 & 10,14 \\
\hline
\end{tabular}

Por lo que podemos inferir que el realizar o no prácticas no es un elemento que aporte valor en términos de calidad de vida. Aspecto que queda corroborado con el ANOva realizado, tanto a nivel de autoevaluación ( $\mathrm{F}=1,90 ; \mathrm{p}=0,1730)$ como a nivel de evaluación externa $(F=1,56 ; p=0,2152)$, con un nivel de confianza del $95 \%$.

No obstante, si en lugar de centrarnos en la realización de prácticas ponemos el foco en la contratación, observamos que las diferencias de puntuación se incrementan a favor de las personas que han conseguido un contrato frente a las que no lo han conseguido (Tabla 9).

\begin{tabular}{|l|l|c|c|c|c|}
\hline \multicolumn{7}{|c|}{ TABLA 9. Contratos e impacto en calidad de vida } \\
\hline \multirow{2}{*}{$\begin{array}{l}\text { Contrataciones } \\
\\
\text { de Vida 2015 } \\
\text { (Autoevaluación) }\end{array}$} & $\begin{array}{c}\text { Índice Calidad } \\
\text { de Vida 2016 } \\
\text { (Autoevaluación) }\end{array}$ & $\begin{array}{c}\text { Índice Calidad } \\
\text { de Vida 2015 } \\
\text { (Evaluador externo) }\end{array}$ & $\begin{array}{c}\text { Índice Calidad } \\
\text { de Vida 2016 } \\
\text { (Evaluador externo) }\end{array}$ \\
\hline \multirow{2}{*}{ Sí } & Media & 118,22 & 112,90 & 116,33 & 117,18 \\
\cline { 2 - 6 } & Desv. típ. & 7,77 & 10,08 & 6,00 & 7,99 \\
\hline \multirow{2}{*}{ No } & Media & 109,28 & 108,86 & 107,60 & 111,13 \\
\cline { 2 - 6 } & Desv. típ. & 11,31 & 10,87 & 13,06 & 10,30 \\
\hline \multirow{2}{*}{ Total } & Media & 110,64 & 109,53 & 108,96 & 112,20 \\
\cline { 2 - 6 } & Desv. típ. & 11,26 & 10,77 & 12,62 & 10,14 \\
\hline
\end{tabular}

Estableciéndose de nuevo diferencias significativas entre las personas que obtienen un contrato y las que no (Ev. Externa: $F=6,36 ; p=0,0140$ y Autoevaluación: $F$ $=7,17 ; \mathrm{p}=0,0094)$, con un $95 \%$ de confianza.

El índice de calidad de vida es una puntuación compuesta de las puntuaciones correspondientes a las ocho dimensiones que propone el modelo. En nuestro estudio hemos querido identificar la posible dependencia entre cada una de las dimensiones y la participación en el programa de Empleo con Apoyo. Para ello hemos usado la variable obtenida de las medias de los datos de 2015 y 2016 en cada una de las dimensiones, excepto Bienestar Físico, ya que entendemos que no tiene especial interés en relación con el empleo. Los resultados obtenidos los mostramos en dos tablas diferentes, la primera de ellas (Tabla 10), correspondiente a las puntuaciones aportadas 
por el evaluador externo y la segunda (Tabla 11), correspondiente a los datos de autoevaluación.

\begin{tabular}{|l|c|c|c|c|c|c|c|}
\hline \multicolumn{2}{|c|}{ TABLA 10. Impacto en dimensiones de calidad de vida y Empleo con apoyo (I) } \\
\hline $\begin{array}{l}\text { Participación } \\
\text { en el programa } \\
\text { de empleo } \\
\text { con apoyo }\end{array}$ & B. Emocional & B. Material & D. Personal & $\begin{array}{c}\text { Relaciones } \\
\text { Interper- } \\
\text { sonales }\end{array}$ & $\begin{array}{c}\text { Inclusión } \\
\text { Social }\end{array}$ & Derechos & $\begin{array}{c}\text { Autodeter- } \\
\text { minación }\end{array}$ \\
\hline Razón-F & 5,33 & 14,30 & 18,38 & 6,89 & 28,62 & 9,45 & 15,69 \\
\hline Valor-p & 0,0216 & 0,0002 & 0,0000 & 0,0091 & 0,0000 & 0,0023 & 0,0001 \\
\hline
\end{tabular}

(Nivel de confianza 95\%).

\begin{tabular}{|l|c|c|c|c|c|c|c|}
\hline \multicolumn{7}{|c|}{ TABLA 11. Impacto en dimensiones de calidad de vida y Empleo con apoyo (II) } \\
\hline $\begin{array}{l}\text { Participación } \\
\text { en el programa } \\
\text { de empleo } \\
\text { con apoyo }\end{array}$ & B. Emocional & B. Material & D. Personal & $\begin{array}{c}\text { Relaciones } \\
\text { Interper- } \\
\text { sonales }\end{array}$ & $\begin{array}{c}\text { Inclusión } \\
\text { Social }\end{array}$ & Derechos & $\begin{array}{c}\text { Autodeter- } \\
\text { minación }\end{array}$ \\
\hline Razón-F & 2,59 & 16,24 & 25,83 & 21,90 & 19,82 & 17,28 & 25,95 \\
\hline Valor-P & 0,1090 & 0,0001 & 0,0000 & 0,0000 & 0,0000 & 0,0000 & 0,0000 \\
\hline \multicolumn{7}{|l|}{ (Nivel de confianza 95\%). }
\end{tabular}

Observamos que en todas las dimensiones evaluadas podemos encontrar una diferencia significativa entre las personas que participan en el programa de empleo frente a las que no participan.

Si analizamos las puntuaciones por cada una de las dimensiones, detectamos que las mayores diferencias entre uno y otro grupo, en función de si evalúa la propia persona o un evaluador externo, se dan en las siguientes dimensiones:

Autoevaluación: Desarrollo Personal, Relaciones Interpersonales, Inclusión Social, Derechos y Autodeterminación.

Evaluador externo: Desarrollo Personal, Inclusión Social y Autodeterminación.

Como se indicaba anteriormente, según Verdugo (2006) el empleo tiene un mayor impacto en dimensiones como Inclusión social, Desarrollo Personal, Autodeterminación y Derechos. Nuestros datos reflejan que las diferencias se establecen fundamentalmente en estas dimensiones y especialmente en las puntuaciones procedentes de la subescala autoadministrada.

A modo de resumen, destacar que observamos diferencias en las puntuaciones obtenidas entre las personas que participan en el programa de empleo con apoyo y las que no. Además, estas puntuaciones se diferencian más en relación a lo que se avance en el programa (participación, realizar prácticas u obtener un contrato). Por último, y teniendo en cuenta nuestros datos, hemos identificado que hay dimensiones concretas en las que se establecen más diferencias: autodeterminación, desarrollo personal, inclusión social y relaciones interpersonales. 


\section{Conclusiones}

En primer lugar, nos gustaría destacar la importancia que desde nuestro punto de vista tiene el uso de las escalas de calidad de vida en el desarrollo del trabajo con personas con discapacidad intelectual, ya que nos permite ir identificando el alcance de la prestación de apoyos en relación con resultados personales. Debido a que a lo largo de estos últimos seis años hemos usado diferentes escalas no hemos podido realizar una comparación válida de los resultados de todos los años. No obstante, hemos detectado que a lo largo del tiempo las personas pueden obtener puntuaciones globales de calidad de vida distintas y no siempre en sentido creciente. Esto nos lleva a pensar que, a la hora de establecer objetivos desde la organización, como por ejemplo "mejorar la calidad de vida de las personas", debemos plantearlos de manera relativa y en periodos amplios de tiempo. Entendemos que sería un error solo tomar la referencia de un año para otro con el objeto de valorar si la vida de la persona ha mejorado o no. Esto nos lleva a plantearnos que tendríamos que pensar en tendencias de puntuaciones a lo largo de periodos amplios de tiempo, por ejemplo, quinquenales, más que en el valor aislado de puntuaciones.

Por otra parte, el índice de calidad de vida es una puntuación que se deriva de las puntuaciones de las ocho dimensiones, por lo que cambios en alguna o algunas de ellas afectarán a la puntuación global. Es por ello que entendemos que no solo hay que mirar a la puntuación global de calidad de vida, sino también analizar por separado cada una de las puntuaciones de las diferentes dimensiones, especialmente atendiendo al enfoque de las diferentes prácticas desarrolladas. No podemos perder de vista que parte de la vida de las personas ocurre al margen de las intervenciones de las organizaciones, por lo que analizar y valorar la influencia de los contextos próximos es un elemento clave a tener en cuenta.

En nuestro estudio nos hemos querido centrar en tres aspectos que consideramos relevantes para una primera aproximación al uso de los datos de calidad de vida: la relación entre necesidades de apoyo y calidad de vida; cómo pueden influir la presencia o no de enfermedad mental, y, por último, qué influencia puede tener un programa concreto de carácter inclusivo como el de empleo con apoyo.

En relación con el primero de los aspectos reseñados hemos podido constatar que hay una relación directa entre las puntuaciones de la Escala sis y las puntuaciones de las escalas de calidad de vida, siendo de tendencia distinta entre las personas evaluadas con la escala Inico-Feaps (relación negativa) y las evaluadas con la Escala San Martín (relación positiva). Puede que en este segundo caso esté teniendo un peso más alto de lo esperado el papel del evaluador externo. No obstante, en un futuro, habría que estudiar este aspecto de manera más detallada. En el caso de las personas con menos necesidades de apoyo los datos indican que se obtienen mejores resultados en calidad de vida a menor intensidad de apoyos.

En relación con la influencia que la salud mental puede tener sobre los resultados de calidad de vida hemos podido constatar que no hay diferencias entre aquellas personas que tienen un diagnóstico de salud mental y las que no. De manera contraria, en el caso de personas a las que se les administra medicación de carácter psiquiátrico y a las que no, podemos constastar que hay diferencias significativas entre ellas en 
términos de resultados de calidad de vida. Es decir, estas últimas obtienen mejores puntuaciones en términos de calidad de vida que las primeras.

Sí hemos podido comprobar que al analizar la dimensión "Bienestar Emocional” se identifican diferencias significativas entre las personas diagnosticadas con trastornos de salud mental y las que no. Es por esto que debe ser un elemento clave a tener en cuenta en los programas y prácticas a desarrollar, ya que si este aspecto no es contemplado de manera específica puede que el impacto de programas convencionales tenga un efecto menor en las personas afectadas por esta situación.

Por último, estábamos interesados en valorar la relación de programas inclusivos con resultados de calidad de vida, en nuestro caso nos hemos centrado en personas que participan en el programa de empleo con Apoyo de Aprosub. En esta ocasión hemos optado por poner el foco en las diferentes dimensiones (excepto bienestar físico) en lugar de en el índice global de calidad de vida. Hemos podido comprobar que, de manera general, las personas que participan en el programa de empleo obtienen mejores puntuaciones en las dimensiones de calidad de vida que las que no participan. Igualmente, en la medida que se avanza en el programa (participación, prácticas o contratos) los resultados mejoran. Por otra parte, tenemos que tener en cuenta que la participación en un programa concreto elegido por la propia persona, como en este caso, supone una alta motivación para su desarrollo, por lo que los logros y la valoración de los mismos se reflejan en sus resultados personales. Además, tendrán un mayor impacto en dimensiones como inclusión social o relaciones interpersonales si los programas o prácticas desarrollados tienen un fuerte carácter inclusivo, como, por ejemplo, el programa de empleo con apoyo.

Queremos finalizar incidiendo en la importancia del uso de mediciones de calidad de vida, ya sea a nivel global o por dimensiones, para conocer el efecto de las prácticas desarrolladas en la vida de las personas. Igualmente, permite obtener una visión global de la orientación de las prácticas de las organizaciones atendiendo a resultados en la calidad de vida de las personas.

\section{Referencias bibliográficas}

Arias, B., Clavero, D., Gómez, L., Santamaría, M., Tamarit, J. y Verdugo, M. Á. (2013). Escala INICO-FEAPS: Evaluación Integral de la Calidad de Vida de personas con Discapacidad Intelectual o del Desarrollo. Salamanca: inico.

Arias, B., Fernández, S., Gómez, L., Hierro, I., Navallas, E., Santamaría, M. y VerDugo, M. Á. (2014). Escala San Martín: Evaluación de la calidad de vida de personas con discapacidades significativas. Santander: Fundación Obra San Martín, inICO.

Arias, B., Gómez, L., Schalock, R. L. y Verdugo, M. Á. (2009). Escala Integral. Evaluación Objetiva y Subjetiva de la Calidad de Vida de Personas con Discapacidad Intelectual. Madrid: CEPE.

Arias, B., IbÁÑEz, A. y Verdugo, M. Á. (2007). La Escala Intensiva de Apoyos (sis): adaptación inicial al contexto español y análisis de sus propiedades psicométricas. Siglo Cero, 38 (2), 3-5.

Benito, M. C., Jordán de Urríes, F. B., Martín, R., Orgaz, B., Ruiz, N., Santamaría, M. y Verdugo, M. Á. (2010). La mejora de la calidad de vida mediante el empleo con apoyo. La experiencia del programa ECA Caja Madrid. Educación y Futuro, 23, 13-30. 
Benito, M., Jordán de Urríes, F. B., Martín, R., Orgaz, B., Santamaría, M. y Verdugo, M. Á. (2012). Influencia de un programa de empleo con apoyo en la calidad de vida percibida de sus usuarios. Siglo Cero, 43 (3), 69-83.

FEAPs (2007). Trastornos de la Salud Mental en las personas con discapacidad intelectual. Madrid: FEAPS.

Luckasson, R., Borthwick-Duffy, S., Buntinx, W. H., Coulter, D. L., Craig, E. M. P., Reeve, A. ... y Tasse, M. J. (2002). Mental retardation: Definition, classification, and systems of supports. Washington, DC: American Association on Mental Retardation.

Luckasson, R., Coulter, D. L., Polloway, E. A., Reiss, S., Schalock, R. S., Shell, M. E. ... y STARK, J. A. (1992). Mental retardation: Definition, classification, and systems of support. Washington, DC: American Association on Mental Retardation.

Martínez-Leal, R., Salvador-Carulla, L. y Salinas, J. A. (2007). Trastornos de la salud mental en personas con discapacidad intelectual: declaración Feaps e informe técnico. Madrid: FEAPS.

Moratalla, C., Ruberte, S. y Villaró, G. (Coords.) (2014). Discapacidad Intelectual y Salud Mental. Evaluación e Intervención Psicológicas. Análisis de casos. Madrid: Plena Inclusión Madrid.

Schalock, R. L. y Verdugo, M. Á. (2003). Calidad de vida: manual para profesionales de la educación, salud y servicios sociales. Madrid: Alianza.

Schalock, R. L. y Verdugo, M. Á. (2013). Discapacidad e Inclusión. Salamanca: Amarú.

Verdugo, M. Á. (Dir.) (2006). Cómo mejorar la calidad de vida de las personas con discapacidad. Instrumentos y estrategias de evaluación. Salamanca: Amarú. 